\title{
TRATAMIENTO DE LA INCONTINENCIA DE ORINA DE ESFUERZO (IUE) FEMENINA MEDIANTE EL EMPLEO DE FASCIA LATA EN UN HOSPITAL RURAL
}

\author{
Federico L. Zeller', Fabio Miani², Laura Pavese3, Jorgelina Sotelano ${ }^{3}$ y Mariela Alvarez³.
}

'Servicio de Urología. Hospital Zonal Ramón Carrillo, Bariloche. Río Negro.

${ }^{2}$ Servicio de Traumatología. Hospital de Área El Bolsón. Río Negro.

${ }^{3}$ Servicio de Ginecología y Obstetricia. Hospital de Área El Bolsón. Río Negro. Argentina.

\begin{abstract}
Resumen.- OBJETIVO: El sling pubovaginal ha sido en los últimos años el tratamiento de elección para la IUE femenina. Los de material autólogo han demostrado el menor índice de complicaciones. Presentamos nuestra experiencia empleando fascia lata autóloga.
\end{abstract}

MÉTODO: Entre mayo de 2005 y julio de 2006 realizamos 10 tratamientos por vía suprapúbica. En 8 casos se ascendieron al espacio retropubiano mediante agujas y en 2 casos se empleó un tunelizador vaginal (Tyco®). En los 8 iniciales se fijaron ambas ramas del sling al ligamento de Cooper. En los 2 restantes se fijaron a la fascia del oblicuo mayor.

\section{$\frac{0}{4}$
$\frac{0}{0}$
$\frac{0}{0}$
$\frac{0}{0}$
$\frac{0}{0}$
$\frac{0}{0}$
0}

Federico L. Zeller

Servicio de Urología

Hospital Zonal Ramón Carrillo 8400, San Carlos de Bariloche Río Negro. (Argentina). zellerfederico@gmail.com fezeller@ciudad.com.ar

Trabajo recibido: 7 de junio 2007.
RESULTADOS: El promedio de edad fue de 57,7 años; el tiempo de seguimiento promedio fue de 14,8 meses $(6-20)$. En 6 casos se corrigió simultáneamente un cistocele. La internación fue de 72 hs. En 2 casos se requirió la sección del sling autólogo debido a hipercorrección. Cuatro pacientes continúan empleando toallas higiénicas, sin embargo las 10 pacientes no refieren IUE. En 4 pacientes el urocultivo posoperatorio fue positivo, con controles negativos. Un caso presentó una candidiasis vaginal.

CONCLUSIÓN: El sling pubovaginal con fascia lata autóloga es un tratamiento efectivo de bajo costo, con una baja tasa de complicaciones.

Palabras clave: Incontinencia de orina de esfuerzo. Sling autólogos. Fascia lata. Incontinencia femenina.

Summary.- OBJECTIVES: Pubovaginal sling has been the treatment of choice for female SUI over the last two years. Those made with autologous materials have demonstrated a lower rate of complications. We present our experience using autologous fascia lata.

METHODS: Between May 2005 and July 2006 we performed 10 procedures through a suprapubic approach. In eight cases they were ascended to the retropubic space using needles, and in 2 cases we employed the vaginal tunneler (Tycoß). In the 8 initial cases both sling branches were anchored to the Cooper's ligament. In the remainder two cases they were anchored to the fascia of the obliquus externus muscle.

RESULTS: Mean age was 57.7 years; mean follow-up time was 14.8 months (6-20). In 6 cases cystocele was simultaneously corrected. Hospital stay was 72 hours. In two cases, section of the autologous sling was required due to excessive correction. Four patients keep 
using pads; nevertheless, all 10 patients refer no SUI. Post-operative urine culture was positive in four patients, with negative controls. One case presented vaginal candidiasis.

CONCLUSIONS: Autologous fascia lata pubovaginal sling is an effective low cost treatment, with a low complication rate.

Keywords: Stress urinary incontinence. Autologous sling. Fascia lata. Female incontinence.

\section{INTRODUCCIÓN}

El uso del sling vaginal como tratamiento para la incontinencia urinaria ha sido empleado por más de 100 años. Actualmente el sling pubovaginal se ha convertido en uno de los procedimientos preferidos para el tratamiento de la incontinencia urinaria de esfuerzo (IUE) femenina $(1,15)$. Las correcciones por vía suburetral fueron descritas en principio por Schultze en 1884 (2), pero el sling suburetral fue publicado en 1907 por Von Giordono, utilizando el músculo gracilis para rodear la uretra $(2,3)$. Price fue el primero en describir el empleo de un sling de fascia en 1933 (20). Alrededor de 1940 Millen, urólogo y Aldridge, ginecólogo, describieron una técnica de sling empleando flaps de fascia de los rectos anteriores del abdomen (17). Con el tiempo la técnica fue sufriendo numerosas modificaciones, tanto técnicas como de materiales, incluyendo fascia de los rectos anteriores del abdomen, tendón de la fascia lata, materiales sintéticos como politetrafluoroetileno, siliconas, polipropileno y poliéster y materiales cadavéricos como fascia lata, duramadre y dermis.

Cada material tiene ventajas y desventajas en su empleo: el material autólogo (fascia lata o recto anterior del abdomen) le agrega tiempo operatorio al procedimiento, riesgo de hematoma, infección de herida, herniación y disconfort temporal para el paciente por presentar otra incisión (16). El mersileno fue el primer material artificial empleado. Se trató de un poliéster, que luego fue discontinuado por la elevada tasa de erosiones que provocaba $(21,22)$.

En lo referente a otros materiales sintéticos la bibliografía reporta complicaciones como erosiones e infecciones; para el caso específico del politetrafluoroetileno con porcentajes superiores al $23 \%$ (4). Dentro de las complicaciones crónicas podemos enunciar fístulas y supuración de heridas (19). Estos eventos adversos fueron los que en primera instancia pusieron en duda el empleo universal de estos materiales. La erosión vaginal se presenta alrededor de las 4 semanas de la cirugía, con dificultad en la cicatrización de la herida vaginal justo sobre la malla (23), mientras que la erosión uretral no ocurre antes de los 8 meses de pos-operatorio (24). En Argentina, Romano y cols. (28) y Tejerizo y cols. (29) han publicado sus experiencias utilizando mallas "artesanales" de polipropileno, con resultados similares a los modelos comerciales sintéticos, pero a un costo inferior. El primer grupo (28) al emplear sling de siliconas $(S m a r t \circledR)$ refieren una tasa de extracción por erosiones del $81,81 \%$ (9/11). El material sintético ideal para la cirugía pélvica aun debe ser desarrollado. Probablemente la biología molecular permita ayudar a definir nuevos materiales sintéticos con menores eventos adversos.

El material cadavérico se ha empleado durante más de 20 años en la práctica quirúrgica, con una respuesta estable y sin complicaciones a lo largo del tiempo (5). El empleo de fascia cadavérica vs. fascia autóloga no muestra diferencias de acuerdo con la bibliografía (6). La gran ventaja del empleo de tejido autólogo es que tiene la mejor biocompatibilidad con el huésped.

Desde diciembre de 2004 hemos realizado en nuestro hospital 14 correcciones de IUE mediante la técnica de sling vaginal; en 10 casos empleamos fascia lata autóloga. Presentamos nuestra experiencia al respecto.

\section{MATERIAL Y MÉTODO}

Entre mayo de 2005 y julio de 2006 realizamos 10 correcciones de IUE empleando fascia lata autóloga como material para el sling pubovaginal. La edad promedio de las pacientes fue de 57,7 años, con un rango entre 39 y 70 años. Todos fueron realizados empleando una sección de fascia lata de $1 \mathrm{~cm}$ de ancho por $20-22 \mathrm{~cm}$. de longitud. Las pacientes se hallaban vírgenes de tratamiento para la IUE. Todas referían incontinencia urinaria frente a la deambulación. Dado que se trató de pacientes que concurren a un hospital de baja complejidad (rural) y escasos recursos, la evaluación previa consistió en una anamnesis, examen físico, examen ginecológico (realizado por LP, JS o MA del Servicio de Ginecología), urocultivo y ecografía renal, vesical y ginecológica. Durante el examen ginecológico se constató en todos los casos la pérdida de orina frente a las maniobras de esfuerzo. Dado que no contamos con la posibilidad de realizar estudios urodinámicos en estas pacientes, evaluamos la función vesical mediante la medición de volumen premiccional y residuo postmiccional por ecografía (éste no debía superar el $15 \%$ del volumen premiccional). 
Para la obtención del sling de fascia lata colocamos a la paciente en posición decúbito dorsal, con un realce en la cadera homolateral al muslo escogido. El sling de fascia lata fue obtenido en todos los casos por uno de los autores (FM), mediante incisión longitudinal en la cara externa del muslo. Se seccionó una porción de fascia lata de $1 \mathrm{~cm}$ de ancho por $20-22 \mathrm{~cm}$. de longitud. A continuación se realizó el cierre de la brecha para evitar la herniación del vasto externo del cuadriceps. Se dejó una venda elástica alrededor del muslo por 7 días. Se colocó luego a la paciente en posición de litotomía y una sonda vesical para reparar la uretra y evacuar la vejiga. Se incidió la cara anterior de la vagina mediante una incisión longitudinal a $1 \mathrm{~cm}$. por debajo del meato urinario. Se disecaron ambos espacios latero-uretrales hasta la fascia endopélvica. En los primeros 8 casos se ascendieron los extremos del sling de fascia lata mediante agujas al espacio retropubiano (Retzius), perforando la fascia endopélvica y se fijaron al ligamento de Cooper homolateral mediante suturas no reabsorbibles, a través de una incisión abdominal paralela al arco pelviano anterior. En los 2 últimos casos se empleó un tunelizador vaginal (Tyco®), para ascender los extremos del sling de fascia lata y se fijaron al orificio de salida de la aponeurosis abdominal. Al sling se le sometió una tensión suficiente para rodear 1 dedo por debajo de la uretra. La sonda vesical se dejó colocada por $72 \mathrm{hs}$.

\section{RESULTADOS}

Hemos tratado 10 pacientes con IUE con una edad promedio de 57,7 años (rango 39-70 años). El tiempo de seguimiento promedio fue de 14,8 meses, con un mínimo de 6 meses y un máximo de 20 meses.

En 6 casos se realizó simultáneamente la corrección de un cistocele. Todas las pacientes permanecieron internadas por $72 \mathrm{hs}$, momento en el cual se les retiró la sonda vesical y se evaluó por un lapso de 12 hs. la calidad de su micción, controlando mediante examen físico la ausencia de globo vesical.

En 2 casos se tuvo que colocar una sonda vesical a las 24 hs. por presentar una retención aguda de orina. Ambas pacientes fueron reoperadas a los 15 días, realizándose la sección del sling vaginal inmediatamente por debajo de la uretra. Todos los casos fueron controlados con cistoscopia. El seguimiento inmediato se realizó con urocultivo a los 7 días de retirada la sonda vesical y luego un urocultivo mensual durante 90 días. En 4 casos tuvimos urocultivos positivos a $E$. Coli en el posoperatorio inmediato, con controles posteriores negativos. A los 30 días de la cirugía se les midió a las pacientes el volumen miccional y el residuo posmiccional (RPM) mediante cateterismo limpio, siendo en 7 pacientes nulo y en las otras 3, en promedio, inferior al $12 \%$ del volumen emitido espontáneamente. Ninguna de las pacientes presentó infección de la herida quirúrgica. Una paciente presentó una candidiasis vaginal a los 20 días de la cirugía, que remitió con el uso de óvulos de cotrimoxazol durante 5 días. El seguimiento mediato consistió en urocultivo y ecografía con medición de RPM cada 6 meses.

A los 6 meses de la cirugía se les realizó a las pacientes 2 preguntas de satisfacción global:

a) ¿Continúa utilizando pañales $\bigcirc$ toallas higiénicas?

b) Si tuviera que definir su estado luego de la cirugía, ¿cuál de las 3 opciones siguientes aplicaría a su situación actual?

1) curada (sin pérdida)

2) mejor (menor pérdida)

3) igual (la pérdida no ha cambiado)

Empleamos en este caso a efectos de una mejor comprensión por parte de las pacientes la palabra "pérdida" como sinónimo de incontinencia. En 4 casos las pacientes referían seguir utilizando toallas higiénicas, sin embargo las 10 pacientes respondieron sentirse curadas. Las preguntas fueron entregadas como cuestionario autoadministrado.

\section{DISCUSIÓN}

La técnica de sling vaginal se comenzó a emplear a comienzos del siglo 20. Inicialmente este procedimiento quedaba reservado para aquellos casos en los cuales el tratamiento quirúrgico para la IUE fallaba (13). Una gran variedad de materiales y técnicas han sido empleados desde entonces, incluyendo politetrafluoroetileno, fascia del recto anterior del abdomen, fascia lata y pared vaginal anterior, entre otros (7-11).

Aldridge fue quien utilizó por primera vez en 1942 la fascia de los rectos anteriores del abdomen como sling pubovaginal (17). Seccionaba 2 "flaps" de fascia de los rectos, los descendía por debajo del cuello vesical y los suturaba entre sí. El empleo de fascia autóloga, tanto fascia de los rectos anteriores del abdomen como fascia lata, ha demostrado tener un elevado porcentaje de éxito (92-95\%) en numerosos estudios (24). En estos estudios no ha sido informada la ocurrencia de erosión uretral con el empleo de fascia autóloga. Blaivas y Jacobs (12) y Mc Guire y 
Lytton (13) popularizaron el empleo de la fascia de los rectos anteriores del abdomen mediante una vía abdomino-vaginal combinada. Las desventajas del empleo de la fascia de los rectos anteriores del abdomen son 3:

a) La presencia de cirugías previas dificulta, por la cicatrización, la toma del sling.

b) Se requieren entre $20-22 \mathrm{~cm}$ de fascia, con los cuales a veces no se cuentan.

c) La extracción de $20-22 \mathrm{~cm}$ de fascia del recto anterior incrementa el tiempo de recuperación del paciente.

El empleo del sling de fascia lata autóloga tiene como desventaja:

a) La cicatriz en la cara lateral del muslo.

b) El tiempo operatorio para su obtención (aproximadamente $45 \mathrm{~min}$.).

Al emplear fascia lata cadavérica se ha estimado que el riesgo de adquirir una infección por virus de inmunodeficiencia adquirida $(\mathrm{VIH})$ es de 1 en $1.667 .600(25)$.

La ventaja del empleo del sling de fascia lata autóloga radica en que el paciente no requiere una recuperación tan prolongada como al emplear fascia de los rectos anteriores del abdomen. La extracción del sling en nuestros pacientes no trajo complicaciones en la deambulación. De hecho la segunda paciente que operamos a los 30 días de la cirugía se reintegró a sus tareas habituales, debiendo recorrer una distancia de $12 \mathrm{~km}$. diarios desde su domicilio hasta su trabajo y regreso en bicicleta. En las primeras 8 pacientes realizamos una incisión abdominal paralela a la arcada pubiana y fijamos los extremos del sling al ligamento de Cooper homolateral. En las 2 últimas pacientes esto no fue necesario, pues al emplear el tunelizador vaginal (Tyco®), fijamos los extremos del sling de fascia lata a través del orificio abdominal a la fascia del oblicuo mayor a cada lado respectivamente.

Es de suma importancia la correcta elección del paciente. Aquellos con síntomas anorrectales significativos $y / o$ con prolapso rectal, requieren de un estudio profundo, incluyendo evaluación de la motilidad anorrectal, previos a la cirugía de corrección. El principio de la cirugía pelviana de reconstrucción se basa en un acabado conocimiento de la anatomía y fisiología del piso perineal y la valoración de los mecanismos involucrados en el correcto funcionamiento del piso perineal, la función de los órganos pélvicos y los espacios pelvianos (26).

Creemos que el secreto en el éxito del sling vaginal radica en la tensión a la cual se somete al mismo, suficiente para coaptar la uretra sin obstruirla. Probablemente sea uno de los momentos más importantes de la cirugía. El sling pubovaginal recupera la anatomía normal de la uretra, al restaurar sus soportes laterales y posterior, provistos normalmente por el ligamento uretropélvico y la fascia periuretral. En cierta medida el sling crea un soporte que re-aproxima la inserción de la fascia periuretral a la pared lateral de la pelvis a la altura de la arcada (ligamento uretropélvico). Esto elimina la hipermovilidad uretral e incrementa la resistencia de la uretra proximal (27).

A largo plazo, el éxito o fracaso del sling pubovaginal, dependería también de la escarificación del mismo, el tipo de sutura empleado para la fijación y eventualmente el sitio de inserción. Al igual que Choe y cols. creemos que la fibrosis de ambos brazos del sling en el espacio de Retzius provoca un efecto de "hormigonado", de modo tal que el sling queda fundido al espacio que lo rodea (18). Probablemente en aquellos slings donde lo que se fija es la sutura y no el brazo del mismo, el éxito dependerá de la tensión y punto de inserción de la sutura. Cuando esta sutura suspendida falla, la IUE reaparece (18).

Otros autores como David-Montefiore y cols. sostienen que el factor preponderante en la evolución posoperatoria inmediata es el uso de slings de material no elástico. En el estudio multicéntrico, prospectivo, randomizado realizado por estos autores no reportan episodios de disuria o RAO posoperatoria (30).

Las pacientes permanecieron internadas durante 3 días con sonda vesical, la cual fue retirada al tercer día pos-operatorio. En todos los casos las pacientes orinaron espontáneamente y fueron dadas de alta ese mismo día por la noche. En 2 casos consultaron a la mañana siguiente por presentar una retención aguda de orina. Se asociaron las mismas a una hipercorrección por tensión excesiva del sling vaginal. Se las trató con una sonda vesical por 15 días, momento en el cual se las re-exploró y se seccionó el sling de fascia lata justo por debajo de la uretra. Ambas pacientes no perdieron capacidad de continencia y continuaron orinando sin dificultad, sin residuo posmiccional medido por ecografía. El seguimiento fue 14,8 meses en promedio y se realizó con urocultivo a los 7 días del alta y luego un urocultivo mensual durante 3 meses. En 4 casos tuvimos urocultivos positivos a $E$. Coli en el posoperatorio inmedia- 
to, con controles posteriores negativos. A los 30 días de la cirugía se les midió a las pacientes el volumen miccional y el residuo posmiccional mediante cateterismo limpio, siendo en 7 pacientes nulo y en las otras 3 inferior al $12 \%$ del volumen emitido espontáneamente. Ninguna de las pacientes presentó una infección de la herida quirúrgica; fueron medicadas con cefalotina $1 \mathrm{~g}$ por vía endovenosa cada 6 hs desde el momento previo a la intervención hasta el alta (3ํㅡ́a pos-operatorio).

Probablemente una de las críticas a nuestro trabajo sea la falta de estudios previos para la correcta evaluación de las presiones vesicales y uretrales, pero debemos tener en cuenta que se trata de una experiencia realizada en el ámbito de un hospital rural, con una población de escasos recursos, donde el empleo de un sling de material sintético no es provisto por su elevado costo. Tampoco podemos utilizar mallas "artesanales" de polipropileno por carecer de óxido de etileno para su esterilización. El empleo del sling de fascia lata brinda una solución para estas pacientes, las cuales mejoran su calidad de vida, reduciendo sus gastos en pañales, perfumes o desodorantes, permitiendo además que se reintegren a su vida laboral.

\section{CONCLUSIONES}

Creemos que el sling pubovaginal con fascia lata autóloga es un tratamiento efectivo y de bajo costo, con una baja tasa de complicaciones, que no requiere de una gran infraestructura tecnológica para su realización. Creemos que cada cirujano debe emplear la técnica de colocación que le sea más afín. Una decisión importante es el tipo de material autólogo a elegir para el sling vaginal: ¿̀debe emplear fascia de los rectos anteriores del abdomen o fascia lata?. La bibliografía demuestra en numerosas series que ambos tipos son de una gran efectividad con una baja morbilidad (14).

La presión ejercida por la industria y la necesidad de disminuir el tiempo quirúrgico han reintroducido el empleo de materiales sintéticos, pero también cada cirujano conoce los riesgos de emplear un cuerpo extraño en proximidad de la uretra, lo cual pone a éstos en duda al momento de elegir el tipo de sling.

El procedimiento quirúrgico ideal debe aumentar la resistencia uretral frente al incremento inesperado de la presión intra-abdominal, sin evitar el descenso normal de la presión intrauretral durante la micción.

\section{BIBLIOGRAFÍA y LECTURAS RECOMENDADAS ( ${ }^{*}$ lectura de interés $y^{* *}$ lectura fundamental)}

**1. Mc GUIRE, E.J.; O'CONNELL, H.E.: "Surgical treatment of intrinsic urethral dysfunction slings". Urol. Clin. North Am. 22: 657, 1995.

2. BARRETT, D.; WEIN, A.J.: "Voiding dysfunction: diagnosis, classification and management". Gillenwater JY, Grayhack JT, Howards ST, Duckett JW eds: Adult and Pediatric Urology, Year Book Medical, Chicago, 863, 1987.

3. HOHENFELLNER, R.; PETRIE, E.: "Sling procedures in surgery". Surgery of Female Incontinence. Editado por SL Stanton y E Tanagho, Springer-Verlag, Berlín, 105, 1986.

4. BENT, A.E.; OSTERGARD, D.R.; ZWICK-ZAFFUTO, M.: "Tissue reaction to expanded polytetrafluoroethylene suburethral sling for urinary incontinence: Clinical and histologic study". Am. J. Obstet. Gynecol., 169: 1198, 1993.

5. COOPER, J.L.; BECK, C.L.: "History of softtissue allografts in orthopedics". Sports Med. Arthrosc. Rev., 1: 2, 1993.

6. MERRITT, W.; PEACOCK, E.E. Jr.; CHVAPIL, M.: "Comparative biology of fascial autografts and allografts". Surg. Forum., 25: 524, 1974.

7. MORGAN, J.E.; FARROW, G.A.; STEWART, F.E.: "The Marlex sling operation for the treatment of recurrent stress urinary incontinence: A 16-year review". Amer. J. Obst. Gynec., 111: 224, 1985.

*8. BECK, R.P.; McCORMICK, S.; NORDSTROM, L.: "The fascia lata sling procedure for treating recurrent genuine stress incontinence of urine". Obst. Gynec., 72: 699, 1988.

9. JUMA, S.; LITTLE, N.A.; RAZ, S.: "Vaginal wall sling: Four years later". Urology, 39: 424, 1992.

10. JEFFCOATE, T.N.A.: "The results of the Aldridge sling operation for stress incontinence". J. Obst. Gynaecol. Brit. Empire, 63: 36, 1953.

11. IOSIF, C.S.: "Porcine corium sling in the treatment of urinary stress incontinence". Arch. Gynecol., 240: 131, 1987.

**12. BLAIVAS, J.G.; JACOBS, B.Z.: "Pubovaginal fascial sling for the treatment of complicated stress urinary incontinence”. J. Urol., 145: 1214, 1991.

*13. McGUIRE, E.J.; LYTTON, B.: "Pubovaginal sling procedures for stress incontinence". J. Urol., 119: 82, 1978.

14. MORGAN, T.O.; WESTNEY, O.L.; McGUIRE, E.J.: "Pubovaginal sling: 4-year outcome analysis and quality of life assessment". J. Urol., 163: $1845,2000$.

*15. LEACH, G.E.; DMOCHOWSKI, R.R.; APPELL, R.A. y cols: "Female stress urinary incontinence clinical guidelines panel summary report on sur- 
gical management of female stress urinary incontinence”. J. Urol., 158: 857, 1997.

16. WRIGHT, J.E.; ISELIN, C.E.; CARR, L.K. y cols.: "Pubovaginal sling using cadaveric allograft fascia for the treatment of intrinsic sphincter deficiency". J. Urol., 160: 759, 1998.

17. ALDRIDGE, A.H.: "Transplantation of fascia for relief of urinary stress incontinence". Am. J. Obstet. Gynecol., 44: 398, 1942.

*18. CHOE, J.M.; KOTHANDAPANI, R.; JAMES, L. y cols.: "Autologous, cadaveric, and synthetic materials used in sling surgery: Comparative biomechanical analysis". Urology, 58: 482, 2001.

19. WEINBERGER, M.W.; OSTERGARD, D.R.: "Long-term clinical and urodynamic evaluation of the polytetrafluoroethylene suburethral sling for treatment of genuine stress incontinence". Obstet. Gynecol., 86: 92, 1995.

20. PRICE, P.B.: "Incontinence of urine and feces". Arch. Surg., 26: 1043, 1933.

21. MOIR, J.C.: "The gauze-hammock operation". J. Obstet. Gynaecol. Br. Commonw., 75: 1, 1968.

22. METRICK, I.; LEE, R.E.: "Delayed transection of urethra by Mersilene tape". Urology, 8: 80, 1976.

23. MYERS, D.L.; LA SALA, C.A.: "Conservative surgical management of mersilene mesh suburethral sling erosion". Am. J. Obstet. Gynecol., 179: 1424, 1998.
**24. KASSARDJIAN, Z.G.: "Sling procedures for urinary incontinence in women". BJU International, 93: 665, 2004.

25. TOMFORD, W.W.: "Transmission of disease through transplantation of musculoskeletal allografts". J. Bone Joint Surg., 77: 1742, 1995.

26. THAKAR, R.; STANTON, S.L.: "Weakness of the pelvic floor: Urological consequences". Hosp. Med., 61: 259, 2000.

27. CESPEDES, R.D.; WINTERS, J.C.; FERGUSON, K.H.: "Colpocleisis for the treatment of vaginal vault prolapse". Tech. Urol., 7: 152, 2001.

*28. ROMANO, S.V.; MARCONI, G.; PONCE, C. y cols.: "Resultados del sling en el tratamiento de la incontinencia femenina en un hospital público". Rev. Arg. de Urol., 71: 167, 2006.

29. TEJERIZO, J.C.; GONZÁLEZ, M.; FAVRE, G. y cols.: "Sling sintético: comparación de resultados entre el uso de diferentes mallas, 10 años de experiencia”. Rev. Arg. de Urol., 69: 228, 2004.

30. DAVID-MONTEFIORE, E.; FROBERT, J.L.; GRISARD-ANAF, M. y cols.: "Peri-Operative Complications and Pain after the Suburethral Sling Procedure for Urinary Stress Incontinence: A French Prospective Randomised Multicentre Study Comparing the Retropubic and Transobturator Routes". European Urology, 49: 133, 2006. 